\title{
Tunable Magnetization Dynamics through Solid-State Ligand Substitution Reaction
}

\author{
Long-Fei Wang, ${ }^{\dagger}$ Jiang-Zhen Qiu, ${ }^{\dagger}$ Jun-Yu Hong, ${ }^{\dagger}$ Yan-Cong Chen, ${ }^{\dagger}$ Quan-Wen Li, ${ }^{\dagger}$ Jian-Hua Jia,${ }^{\dagger}$ Jesús Jover, ${ }^{\ddagger}$ Eliseo \\ Ruiz, ${ }^{*}$ Jun-Liang Liu, ${ }^{* \dagger}$ and Ming-Liang Tong**
}

"Key Lab of Bioinorganic and Synthetic Chemistry of Ministry of Education, School of Chemistry, Sun Yat-Sen University, Guangzhou 510275, P. R. China

tDepartament de Química Inorgànica i Orgànica and Institut de Recerca de Química Teòrica i Computacional, Universitat de Barcelona, Diagonal 645, E-08028 Barcelona, Spain

Supporting Information Placeholder

ABSTRACT: The dimeric molecule $\left[\mathrm{Dy}_{2}(\mathrm{acac})_{6}(\mathrm{MeOH})_{2}(\mathrm{bpe})\right] \cdot \mathrm{bpe} \cdot 2 \mathrm{MeOH} \quad(\mathbf{1}$, acac $=$ acetylacetonate, bpe $=$ 1,2-bis(4-pyridyl)ethylene) undergoes a solid-state ligand substitution reaction upon heating, leading to the one-dimensional chain $\left[\mathrm{Dy}(\mathrm{acac})_{3}(\mathrm{bpe})\right]_{n}(\mathbf{2})$. This structural transformation takes advantage of the potential coordination of the guest bpe molecules present in 1. In both complexes the Dy(III) ions adopt similar octacoordinated $D_{4 \mathrm{~d}}$ geometries. However, the different arrangement of the negatively charged and neutral ligands alters the direction of magnetic anisotropy axis and the energy states, thus resulting in largely distinct magnetization dynamics, as revealed by the CASSCF/RASSI calculations.

\section{INTRODUCTION}

Single-molecule magnets ${ }^{1,2}$ (SMMs) bearing magnetic bistability and exhibiting slow relaxation of magnetization at molecular level have become the focus of considerable research efforts due to their potential applications in ultrahigh-density storage and quantum computation. ${ }^{3}$ Such super-paramagnetic behavior origins from the combination of strong magnetic anisotropy and large spin multiplicity of the ground state. This leads to a high energy barrier that hampers the reversal of the molecular spin, which results in a slow magnetic relaxation between "spin up" and "spin down" states. Lanthanide (Ln) ions, like Dy(III), have become the ideal candidates for researchers to construct SMMs due to their inherent large magnetic anisotropy derived from their large angular momentum quantum number $(J){ }^{4}$ The interaction between the lanthanide center and the surrounding atoms leads to the zero-field splitting of the spin-orbit coupled ground state $\left({ }^{2 \mathrm{~S}+1} \mathrm{~L}_{\mathrm{J}}\right)$. This splitting has been demonstrated to be very sensitive to the crystal field in which the magnetic ion resides. The repulsive interaction between the aspherical electron density distribution of the metal and the electrostatic potential of the surrounding ligands has been proved to be the key factor for determining the energy levels, eigenstate composition and the direction of magnetic anisotropy axis of the Ln(III) ions. ${ }^{5 \mathrm{a}-\mathrm{d}}$ Moreover, a recent study on an Er-trensal complex indicates that covalency effects can also play an important role in determining low-lying energy spectrum and $g$ factors of the ground Kramers doublet. ${ }^{5 e}$ Manipulation of the coordination sphere in lanthanide-based SMMs has been successfully utilized for tuning molecular magnetism thanks to the strong correlations between crystal field and magnetization dynamics. ${ }^{6}$ For example, the change of counter-anions in some distorted- $D_{4 \mathrm{~d}}$ mononuclear Dy(III) complexes results in a cis-trans isomerism of the chelate ligands, thus altering the magnetic behavior. ${ }^{6 a}$ In a second example, the replacement of one porphyrin pyrrole nitrogen atom by oxygen or sulfur in bis(tetrapyrrole) Dy(III) complexes results in enhanced energy barriers and in the appearance of butterfly-shaped hysteresis loops. ${ }^{6 \mathrm{~b}}$ (a)

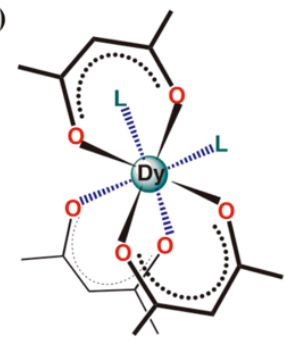

(c)

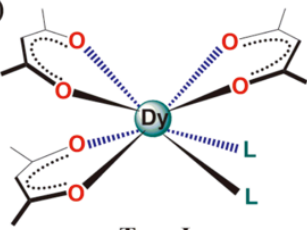

(b)

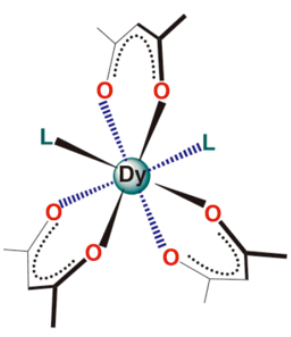

(d)

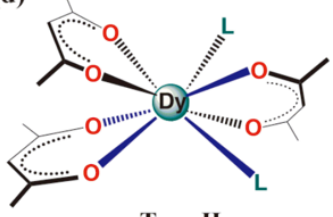

Type II

Scheme 1 Schematic drawing of the $D_{4 \mathrm{~d}}$ coordination of Dy(III) ions with top (up) and side (bottom) views for the Type I (a, c) and Type II $(\mathrm{b}, \mathrm{d})$ configurations of the $\left[\mathrm{Dy}(\mathrm{acac})_{3}(\mathrm{~L})_{x}\right]$ system ( $\mathrm{L}=$ neutral auxiliary ligands).

In 2010 Gao et al. reported a $\left[\mathrm{Dy}(\mathrm{acac})_{3}\left(\mathrm{H}_{2} \mathrm{O}\right)_{2}\right]$ mononuclear complex with SMM behavior that presented a butterfly-shaped hysteresis loop. ${ }^{7}$ Since then, many similar Dy(III) $\beta$-diketone SMMs, with distorted $D_{4 \mathrm{~d}}$ geometries (Type I in Scheme 1), have been prepared and their magneto-structural correlations, in which the neutral capping ligand has been replaced by other moieties, have been studied $^{8}$ 


\begin{abstract}
We report herein the successful transformation of a Dy(III) $\beta$-diketone system from a Type I complex into a Type II analogue (Scheme 1) by a solid-state ligand substitution reaction, which produces an alteration of the coordination sphere of the metal center. The substitution of the coordinated $\mathrm{MeOH}$ molecules around Dy(III) ions by be guests not only leads to the structural transformation from a dimeric Dy(III) complex, $\left[\mathrm{Dy}_{2}(\mathrm{acac})_{6}(\mathrm{MeOH})_{2}(\mathrm{bpe})\right] \cdot$ bpe$2 \mathrm{MeOH}(\mathbf{1})$, to a $\left[\mathrm{Dy}(\mathrm{acac})_{3}(\mathrm{bpe})\right]_{n}$ chain (2), but also causes the rearrangement of the coordination atoms around the Dy(III) ions, consequently changing the crystal field and modulating the magnetization dynamics.
\end{abstract}

\section{EXPERIMENTAL SECTION}

Materials and General Procedures. All reactions and manipulations described below were performed under aerobic conditions. Metal salts were commercially available and used as received without further purification. Magnetic susceptibility measurements were collected using a Quantum Design MPMS XL-7 SQUID magnetometer and a Quantum Design PPMS with VSM option. Polycrystalline samples were embedded in vaseline to prevent torqueing. AC magnetic susceptibility data measurements were performed with a $5 \mathrm{Oe}$ ac field at frequencies between 0.1 and $1488 \mathrm{~Hz}$. Magnetic hysteresis loops were continuously measured on VSM with the field ramping rate of $200 \mathrm{Oe} / \mathrm{s}$. All data were corrected for the diamagnetic contribution calculated using the Pascal constants. The $\mathrm{C}$ and $\mathrm{H}$ microanalyses were performed on freshly-filtered samples, which were taken out immediately from mother liquor, with an Elementar Vario-EL CHN elemental analyzer. X-ray powder diffraction (XRPD) intensities for polycrystalline samples were measured on a Bruker D8 Advance Diffractometer $\left(\mathrm{Cu} K_{\alpha}, \lambda\right.$ $=1.54056 \AA$ ). The thermogravimetric (TG) analyses were performed on a NETZSCH TG209F3 thermogravimetric analyzer in flowing $\mathrm{N}_{2}$ with a heating rate of $10 \mathrm{~K} / \mathrm{min}$.

Synthesis. $\left[\mathrm{Dy}_{2}(\mathrm{acac})_{6}(\mathrm{bpe})(\mathrm{MeOH})_{2}\right] \cdot \mathrm{bpe} \cdot 2 \mathrm{MeOH}(\mathbf{1})$. A mixture of Dy(acac $)_{3}(0.1 \mathrm{mmol})$ and bpe $(0.2 \mathrm{mmol})$ was dissolved in $0.5 \mathrm{~mL} \mathrm{MeOH}$. After stirring for about 10 minutes, the resulting suspension was filtered. The filtrate was sealed in a $5 \mathrm{~mL}$ glass tube. Colourless block crystals of 1 suitable for X-ray analysis were obtained for about several hours at $5{ }^{\circ} \mathrm{C}$. Yield: $76 \%$ based on Dy(acac) $)_{3}$. Elemental analysis calcd (\%) for $\mathrm{C}_{44} \mathrm{H}_{60} \mathrm{Dy}_{2} \mathrm{~N}_{2} \mathrm{O}_{14}: \mathrm{C}: 49.33, \mathrm{H}: 5.57, \mathrm{~N}$ : 3.97; found (\%): C: 49.43, H: 5.36, N: 3.72 .

$\left[\mathrm{Dy}(\mathrm{acac})_{3}(\mathrm{bpe})\right]_{n}$ (2) A mixture of Dy $(\mathrm{acac})_{3}(0.1 \mathrm{mmol})$ and bpe $(0.1 \mathrm{mmol})$ was dissolved in $0.5 \mathrm{~mL} \mathrm{MeOH}$. After stirring for about 10 minutes, the resulting suspension solution was filtered. The filtrate was layered by $0.5 \mathrm{~mL}$ isopropanol solution with $0.07 \mathrm{mmol}$ bpe and then was sealed in a $5 \mathrm{~mL}$ glass tube. Colourless plate crystals of 2 suitable for X-ray analysis were obtained after several hours at $5{ }^{\circ} \mathrm{C}$. Yield: $69 \%$ based on Dy(acac) $)_{3}$. Elemental analysis calcd (\%) for $\mathrm{C}_{27} \mathrm{H}_{31} \mathrm{DyN}_{2} \mathrm{O}_{6}$ : C: $50.51, \mathrm{H}: 4.87, \mathrm{~N}: 4.36$; found (\%): C: 50.41, H: 4.94, N: 4.42.

As may be observed the Dy:bpe ratio (1:1) is the same in both complexes $\mathbf{1}$ and $\mathbf{2}$. However, the amount of bpe in both reactions is higher $(1: 2)$ and varies: 2 equivalents of bpe are employed in the synthesis of 1 while 1.7 ( 1 in the first step plus 0.7 in the isopropanol layer) equivalents of bpe are used to obtain complex 2 . bpe is always added in excess in order to enforce its coordination and also to compensate the high oxygenphilic character of dysprosium. As may be observed, the nature of the solvent employed for crystallization seems to have a great impact in the final products; the $\{\mathrm{Dy}\}_{n}$ chain (2) is obtained in isopropanol probably due to the lower polarity of this solvent, which facilitates the rearrangement of the neutral bpe ligand to fully act as a bridge between two dysprosium atoms.

X-ray Crystallography. Diffraction data for complex $\mathbf{1}$ was collected on a Bruker D8 QUEST diffractometer using $\operatorname{Mo} K_{\alpha}$ radiation $(\lambda=0.71073 \AA)$ at $150 \mathrm{~K}$. Complex 2 was recorded on an Oxford Diffraction Gemini R CCD diffractometer with $\mathrm{Cu} K_{\alpha}, \lambda=1.54056 \AA$ at $150 \mathrm{~K}$. The structures of all complexes were solved by direct methods, and all non-hydrogen atoms were refined anisotropically by least-squares methods on $F^{2}$ using the SHELXTL program. Hydrogen atoms on organic ligands were generated in idealized positions and refined using a riding model. Data has been deposited at the Cambridge Structural Database (CSD) with the following CCDC numbers: $\left[\mathrm{Dy}_{2}(\mathrm{acac})_{6}(\mathrm{bpe})(\mathrm{MeOH})_{2}\right] \cdot \mathrm{bpe} \cdot 2 \mathrm{MeOH}(\mathbf{1}), \mathrm{CCDC}-1051853$ and $\left[\mathrm{Dy}(\mathrm{acac})_{3}(\mathrm{bpe})\right]_{n}(2), \mathrm{CCDC}-1051854$. Crystal data and structural refinements are listed in Table 1.

Table 1. Crystallographic Data and Structural Refinements for $\mathbf{1}$ and $\mathbf{2}$.

\begin{tabular}{|c|c|c|}
\hline Compound & 1 & 2 \\
\hline Empirical formula & $\mathrm{C}_{58} \mathrm{H}_{78} \mathrm{Dy}_{2} \mathrm{~N}_{4} \mathrm{O}_{16}$ & $\mathrm{C}_{27} \mathrm{H}_{31} \mathrm{DyN}_{2} \mathrm{O}_{6}$ \\
\hline Formula weight & 1412.24 & 642.04 \\
\hline Temperature / K & 150 & 150 \\
\hline Crystal system & Triclinic & Monoclinic \\
\hline Space group & $P-1$ & $P 2 / c$ \\
\hline$a / \AA$ & $9.2051(5)$ & $8.0500(3)$ \\
\hline$b / \AA$ & $10.0965(6)$ & $8.5931(3)$ \\
\hline$c / \AA$ & $16.8051(9)$ & $19.5976(6)$ \\
\hline$\alpha /{ }^{\circ}$ & $98.424(2)$ & 90 \\
\hline$\beta /{ }^{\circ}$ & $92.981(2)$ & $98.154(3)$ \\
\hline$\gamma / 0$ & $100.517(2)$ & 90 \\
\hline$V / \AA^{3}$ & $1514.06(15)$ & $1341.95(8)$ \\
\hline$Z$ & 1 & 2 \\
\hline$\rho_{\text {calc }} /$ g.cm ${ }^{-3}$ & 1.549 & 1.589 \\
\hline$F(000)$ & 714.0 & 642.0 \\
\hline Crystal size $/ \mathrm{mm}^{3}$ & $0.16 \times 0.11 \times 0.07$ & $0.20 \times 0.19 \times 0.18$ \\
\hline Goodness-of-fit on $F^{2}$ & 1.096 & 1.054 \\
\hline Final $R$ indices $[I \geq 2 \sigma(I)]^{\mathrm{a}}$ & $\begin{array}{l}R_{1}=0.0238, w R_{2}= \\
0.0493\end{array}$ & $\begin{array}{l}R_{1}=0.0404, w R_{2}= \\
0.1030\end{array}$ \\
\hline Final $R$ indices [all data] ${ }^{\mathrm{b}}$ & $\begin{array}{l}R_{1}=0.0296, w R_{2}= \\
0.0505\end{array}$ & $\begin{array}{l}R_{1}=0.0419, w R_{2}= \\
0.1059\end{array}$ \\
\hline \multicolumn{3}{|c|}{${ }^{\mathrm{a}} R_{1}=\sum|| F_{\mathrm{o}}|-| F_{\mathrm{c}}|| / \sum\left|F_{\mathrm{o}}\right|,{ }^{\mathrm{b}} w R_{2}=\left[\sum w\left(F_{\mathrm{o}}{ }^{2}-F_{\mathrm{c}}{ }^{2}\right)^{2} / \sum w\left(F_{\mathrm{o}}{ }^{2}\right)^{2}\right]^{1 / 2}$} \\
\hline
\end{tabular}

Computational details. $A b$ initio $\operatorname{CASSCF}(7,9)$ calculations (including 21, 128 and 98 states for sextet, quartet and doublet multiplicities, respectively) were carried out for complexes 1 and 2 using the Molcas $8.0^{10}$ package. In both cases the structures were reduced to mononuclear Dy(III) complexes like the ones shown in Figure 1a,b, including all the methanol, bpe and $\mathrm{acac}^{-}$ligands directly attached to the metal center. The all electron ANO-RCC basis set ${ }^{11}$ was employed in these calculations, including the following contractions: Dy [9s8p6d4f3g2h]; O [4s3p2d1f]; N [4s3p2d1f] and [3s2p] for atoms directly attached or far away from the Dy center, respectively; C [3s 2p] and H [2s]. 


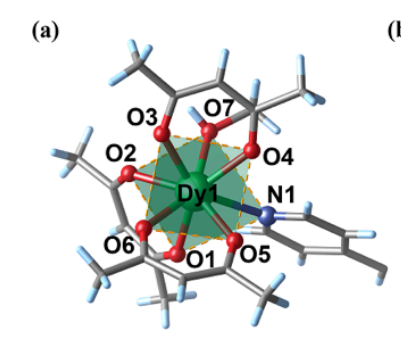

(b)
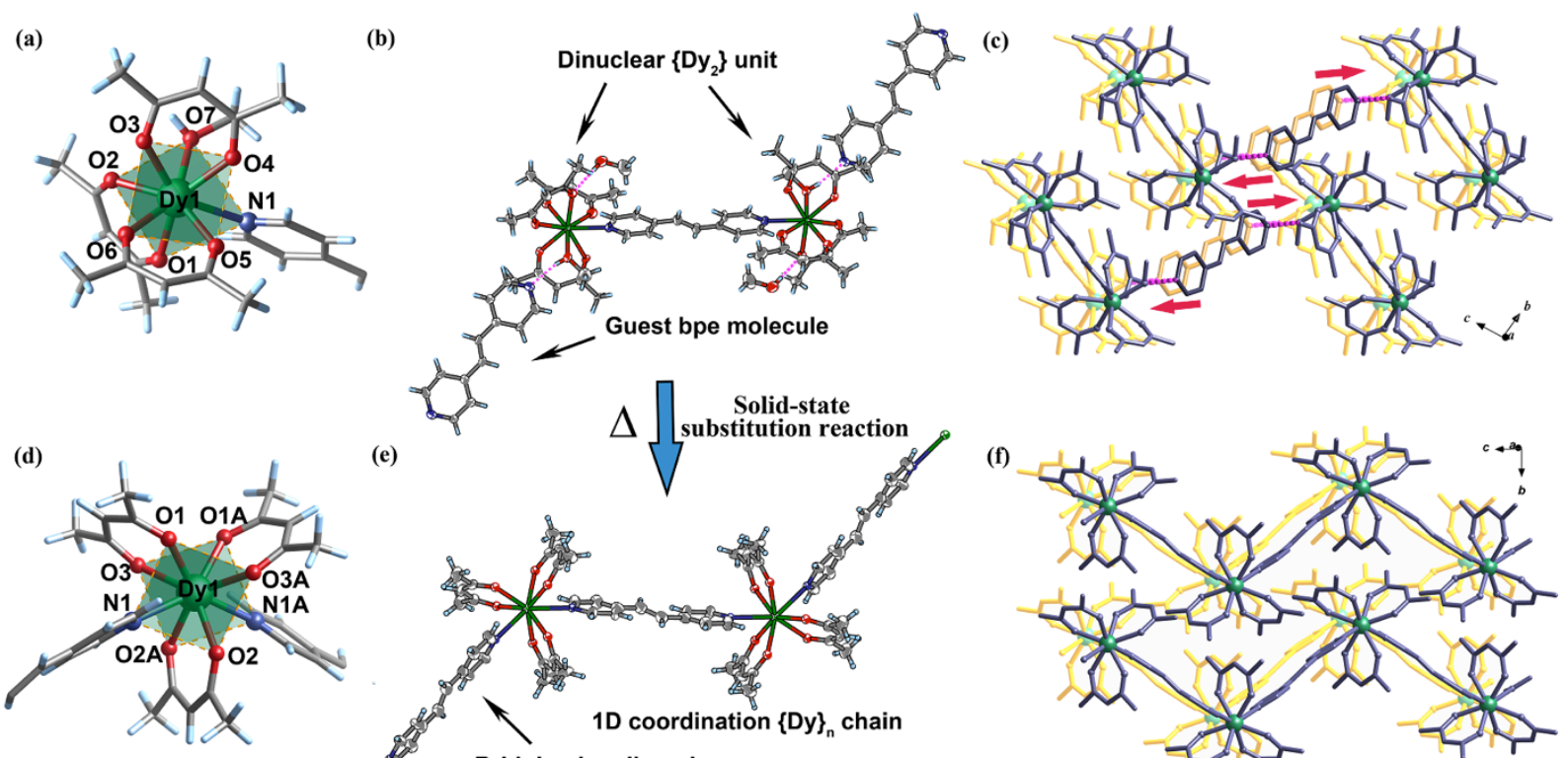

(e)
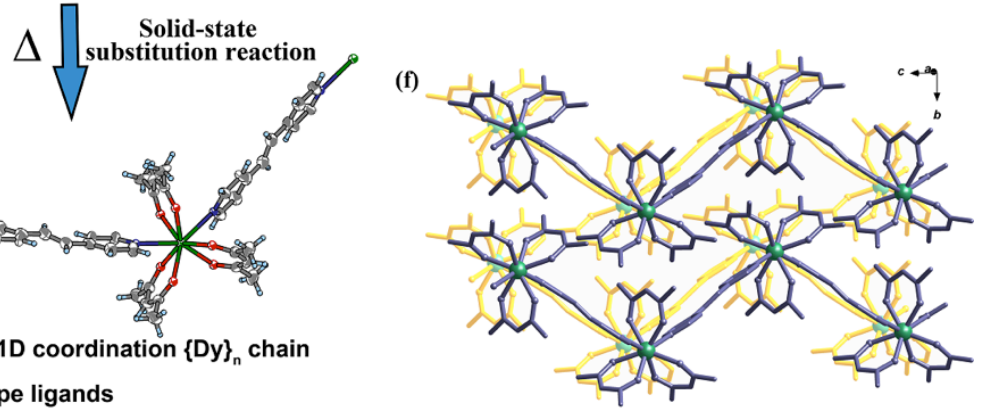

Figure. 1 The coordination environment of Dy(III) in complex 1 (a) and 2 (d), respectively. Color Codes: Dy, green; N, blue; O, red; C, gray; $\mathrm{H}$, pale blue. Symmetry code: (A) $-1-x, y, 3 / 2-z$. A view of the dinuclear $\left\{\mathrm{Dy}_{2}\right\}$ unit and the hydrogen-bonded bpe molecules in 1 (b) and a segment of the $\{\mathrm{Dy}\}_{n}$ chain in $\mathbf{2}$ (e). The packing diagrams of the dinuclear $\left\{\mathrm{Dy}_{2}\right\}$ units and the guest bpe molecules for $\mathbf{1}$ (c) and the 1D $\{\mathrm{Dy}\}_{n}$ coordination chains for $\mathbf{2}$ (f) along $a$ axis. The red arrows are the potential coordination direction of guest bpe molecules. The hydrogen bonds are displayed as purple dashed lines.

The spin-orbit coupling has been introduced as implemented in the SO-RASSI (Restricted Active Space State Interaction) approach and the magnetic properties were obtained with the SINGLE_ANISO code). ${ }^{12}$

\section{RESULTS AND DISCUSSION}

Crystal Structure. The single-crystal X-ray crystallography data (Table 1 ) reveals that $\mathbf{1}$ crystallizes in the $P-1$ space group and contains one crystallographically independent Dy(III) ion in the asymmetric unit. In complex $\mathbf{1}$, the Dy(III) ion (Figure 1a) is eight-coordinate $\left(\mathrm{NO}_{7}\right)$ with a distorted square antiprism coordination geometry by three chelated $\mathrm{acac}^{-}$, one bpe ligand and one methanol molecule. Four $\mathrm{O}$ atoms from two acac ions serve as one square while the remaining acac ion, the $\mathrm{O}$ atom of methanol and the bpe ligand offer three $\mathrm{O}$ atoms and one $\mathrm{N}$ atom for the other square. Such square antiprism coordination sphere of Dy(III) center in 1 (Type I in Scheme 1) is similar to some other reported complexes with general formula $\left[\mathrm{Dy}(\mathrm{acac})_{3}(\mathrm{~L})_{x}\right]^{7,8}$ (where L can be mono- or bi-dentate with $x=2$ or 1 , respectively). A further $S H A P E^{13}$ analysis (Table 2) gives a square antiprism $C S h M$ value of 0.827 for 1 , indicating the magnitude of deviation from the ideal $D_{4 \mathrm{~d}}$ symmetry. Each coordinated bpe ligand bridges two Dy(III) ions through its two terminal $\mathrm{N}$ atoms, producing the corresponding dinuclear $\left\{\mathrm{Dy}_{2}\right\}$ units (Figure $1 \mathrm{~b}$ ) with a Dy $\cdots$ Dy distance of $14.71 \AA$. The guest bpe molecules are anchored through the complementary $\mathrm{N} \cdots \mathrm{H}-\mathrm{OMe}$ hydrogen bonds with the coordinated methanol molecules (Figure 1c).
Table 2. The CShM values calculated by SHAPE $2.1^{13}$ for the Dy(III) ions in $\mathbf{1}$ and 2 . $^{\mathrm{a}}$

\begin{tabular}{lll}
\hline Complex & SAPR-8 $\left(D_{4 d}\right)$ & TDD-8 $\left(\boldsymbol{D}_{\mathbf{2 d}}\right)$ \\
$\mathbf{1}$ & 0.827 & 1.217 \\
$\mathbf{2}$ & 0.501 & 2.536
\end{tabular}

${ }^{a}$ SAPR- $8=$ Square antiprism; TDD- $8=$ Triangular dodecahedron

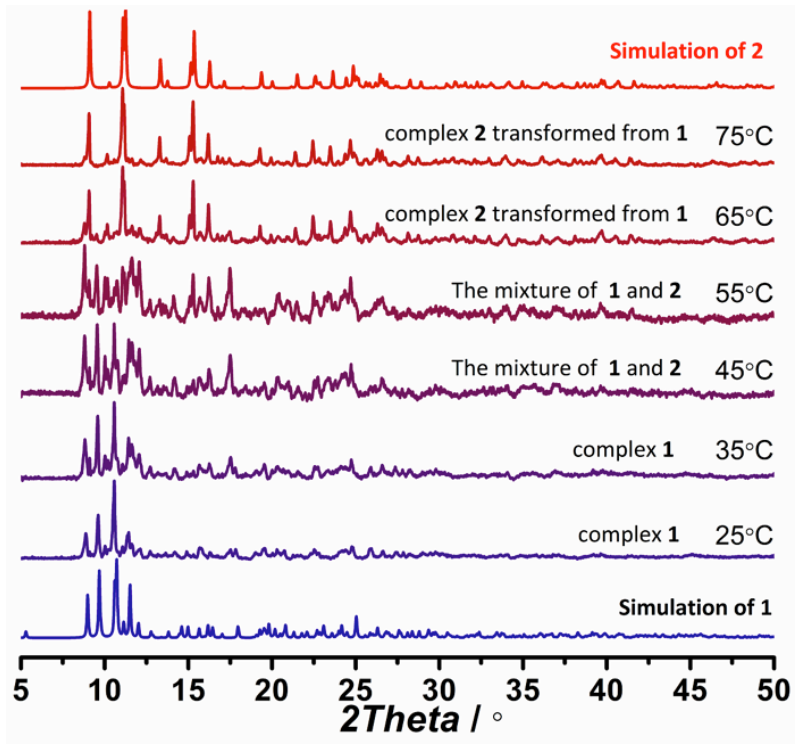

Figure 2 The solid-state transformation from 1 to $\mathbf{2}$ monitored by VT-PXRD at different temperatures. 
Considering the coordination potential of the guest bpe molecules and that they have free nitrogen donor atoms, we speculated a solid-state ligand substitution reaction, where the methanol ligand could be replaced by bpe.This kind of reaction has already been observed in some flexible frameworks ${ }^{14}$ with specific oriented host-guest interactions. The thermogravimetry (TG) and the variable-temperature powder X-ray diffraction (VT-PXRD) were measured to probe such solid-state ligand replacement. Upon heating, complex 1 suffers a mass loss of $9.42 \%$ at about $60{ }^{\circ} \mathrm{C}$ (Figure S2), which agrees with the complete desolvation of the coordinated and the guest $\mathrm{MeOH}$ molecules (9.07\%). Moreover, the VT-PXRD further indicates the crystal transformation from 1 to a new complex above $65{ }^{\circ} \mathrm{C}$ (Figure 2). Although the single crystallinity of $\mathbf{1}$ was lost during the structural transformation, luckily, through the change of synthetic condition (See experimental section), the single crystal of complex 2 (Table 1) suitable for single-crystal X-ray diffraction was obtained. The PXRD experiment (Figure $\mathrm{S} 1$ and 2) confirms that the as-synthesized complex $\mathbf{2}$ and the TG transformed powder are identical.

As expected, the single-crystal structure of 2 shows that the terminal methanol ligand has been replaced by the hydrogen-bonded bpe molecules upon heating, thus confirming thesolid-state ligand substitution. After the coordination of the guest bpe molecules, the $\left\{\mathrm{Dy}_{2}\right\}$ units in $\mathbf{1}$ are connected with each other, resulting in the one-dimensional coordination chain of $\mathbf{2}$ (Figure 1e). In $\mathbf{2}$, each Dy(III) ion is coordinated to six $\mathrm{O}$ atoms from three chelating acac and two $\mathrm{N}$ atoms from two bpe ligands (Figure 2d), leading again to a distorted $D_{4 \mathrm{~d}} \quad\left(\mathrm{~N}_{2} \mathrm{O}_{6}\right)$ coordination sphere. The square antiprism CShM value of Dy(III) in 2 is 0.501 (Table 2), which is smaller than that of $\mathbf{1}$, indicating that the coordination geometry of the Dy(III) center in $\mathbf{2}$ is closer to the reference $\left(D_{4 \mathrm{~d}}\right)$ shape. Moreover, and due to the coordination of the guest bpe from 1 to 2 , the steric effect prompts the cooperative rearrangement of the other ligands, leading to a change of the coordination sphere (Scheme 1). As the rearrangement of the negatively charged and the neutral ligands would undoubtedly change the crystal field of Dy(III) ion, it is interesting to investigate its influence on magnetization dynamics from $\mathbf{1}$ to 2 .

Magnetic Properties. The temperature-dependent magnetic susceptibilities were recorded in a direct-current (dc) field of $1 \mathrm{kOe}$ (Figure 3). At room temperature, $\chi_{\mathrm{M}} T$ values of $13.8 \mathrm{~cm}^{3} \mathrm{~K} \mathrm{~mol}^{-1}$ are found for both 1 and 2 , which are slightly smaller than the expected value of one Dy(III) ion $\left(14.2 \mathrm{~cm}^{3} \mathrm{~K} \mathrm{~mol}{ }^{-1}\right.$ for the ${ }^{6} H_{15 / 2}$ state). This could be ascribed to the fact that the ground Russell-Saunders multiplet is not equally populated even at room temperature. Upon cooling, the $\chi_{\mathrm{M}} T$ values of $\mathbf{1}$ and $\mathbf{2}$ gradually decrease to 11.1 and $9.7 \mathrm{~cm}^{3} \mathrm{~K} \mathrm{~mol}^{-1}$, respectively, indicating the further depopulation of Stark sublevels of Dy(III) ion and/or dipolar magnetic interactions. The magnetization at $2 \mathrm{~K}$ increases rapidly with the magnetic field strength up to about $9 \mathrm{kOe}$ and then increases slowly, finally reaching values of $5.22 N \beta$ for $\mathbf{1}$ and $5.93 N \beta$ for $\mathbf{2}$ at $70 \mathrm{kOe}$ (Figure S3). Detailed alternating-current (ac) susceptibility measurements were also carried out on the single crystal samples of $\mathbf{1}$ and $\mathbf{2}$ to study their dynamic behavior.

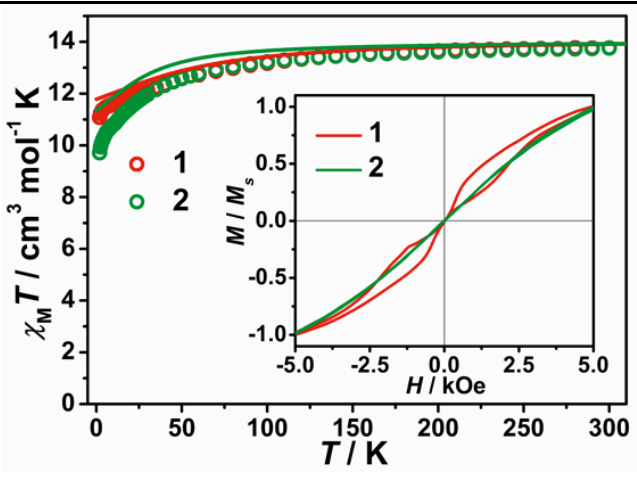

Figure 3 Temperature dependent $\chi_{\mathrm{M}} T$ products at $1 \mathrm{kOe}$ for $\mathbf{1}$ (red) and 2 (green). The solid lines correspond to the ab initio calculations. Inset: plot of magnetization versus field for $\mathbf{1}$ (red) and 2 (green) at $2 \mathrm{~K}$, respectively, with a sweep rate of $200 \mathrm{Oe}$ $\mathrm{s}^{-1}$.
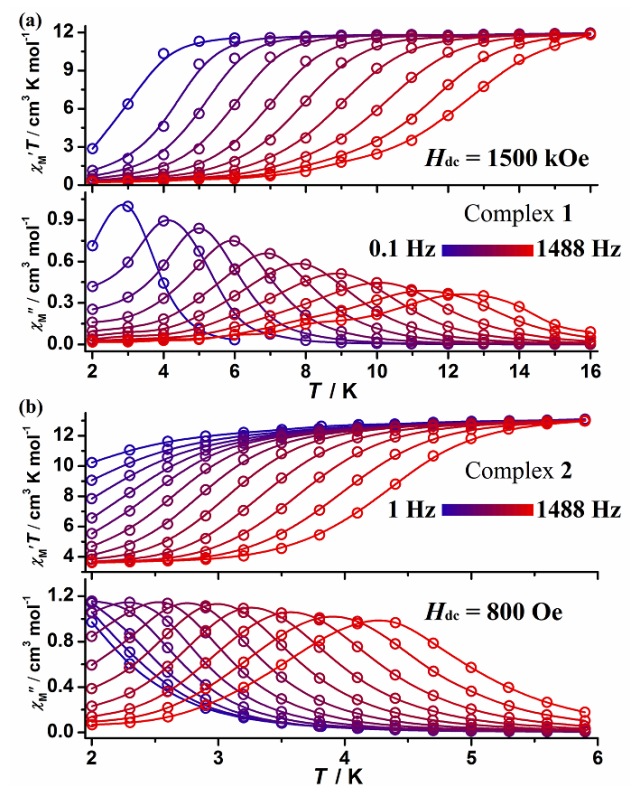

Figure 4 The temperature-dependent ac susceptibilities for $\mathbf{1}$ at a 1500 Oe (a) dc fields, and $\mathbf{2}$ under an 800 Oe dc field (b).

At zero field, the out-of-phase $\left(\chi_{\mathrm{M}}{ }^{\prime \prime}\right)$ signals for both complexes were not observed due to the fast quantum tunneling of magnetization (QTM) which could arise from crystal-field effect, hyperfine interaction and dipolar interactions.

A series of external dc fields in the range of 0.2-5.0 kOe were applied to suppress the QTM process in 1 at $4 \mathrm{~K}$ and 2 at $2 \mathrm{~K}$. As a result, a set of peaks of $\chi_{\mathrm{M}}$ " signals at various fields were observed in the range of $0.1-1488 \mathrm{~Hz}$ for both complexes (Figure S4), indicating the decline of relaxation processes. The optimized dc fields are 1500 Oe for 1 and 800 Oe for 2 (Figure S5), respectively. The temperature-dependent ac susceptibility measurements (Figure 4) at the optimized dc fields for $\mathbf{1}$ and $\mathbf{2}$ were performed. No "tails" of the out-of-phase $\chi_{\mathrm{M}}$ " signals were observed for none of complexes at low temperature, suggesting the suppression of QTM. At the ac frequency of $1488 \mathrm{~Hz}$, the peaks of $\chi_{\mathrm{M}}$ " signals for 1 were observed at 12.5 K, higher than that of $\mathbf{2}$, which were obtained at a temperature of $4.3 \mathrm{~K}$. 


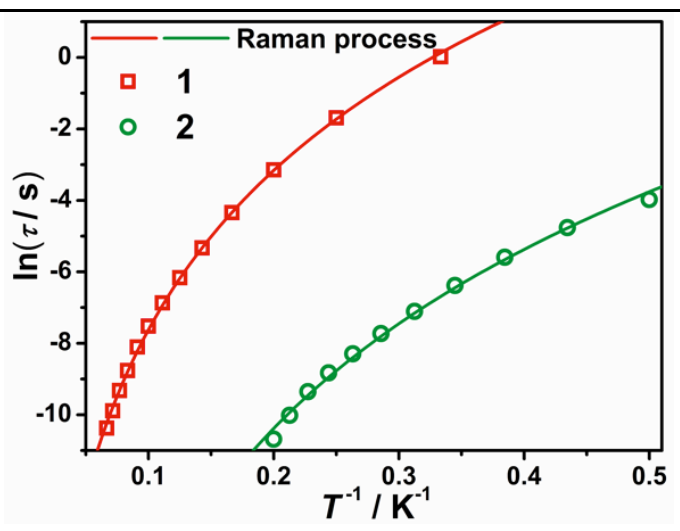

Figure 5 The $\ln \tau$ vs $T^{-1}$ plots for 1 (red) and $\mathbf{2}$ (green) at 1500 and 800 Oe fields, respectively. The solid lines represent the Raman process for $\mathbf{1}$ and $\mathbf{2}$ with a $\tau^{-1} \sim T^{n}$ law.

On the other hand, the frequency-dependent ac susceptibility (Figure S6), measured at $2 \mathrm{~K}$, shows that the peaks of $\chi_{\mathrm{M}}$ " signals for $\mathbf{1}$ are located at the frequency region below $1 \mathrm{~Hz}$ while those of $\mathbf{2}$ are located at $10.8 \mathrm{~Hz}$, suggesting that the relaxation time for $\mathbf{1}$ is much longer than that for $\mathbf{2}$ at low temperature region. The butterfly-shaped hysteresis loop of $\mathbf{1}$ at $2 \mathrm{~K}$ further confirms the slower relaxation process than that of $\mathbf{2}$ under an external field (Figure 3 inset). The relaxation time $(\tau)$ of both complexes was also extracted by fitting the ac magnetic susceptibilities with a generalized Debye model (Figure S6b, d). The resulting distribution coefficient values $(\alpha)$ for $\mathbf{1}(>5 \mathrm{~K})$ and $2(>3 \mathrm{~K})$ are in the range of $0-0.2$, indicating the narrow-to-moderate distribution of slow magnetic relaxation. However, the $\alpha$ values increase to 0.38 for 1 (at $3 \mathrm{~K}$ ) and 0.33 for 2 (at $2 \mathrm{~K}$ ); these relatively large $\alpha$ values for both complexes at low temperature region may be attributed to the onset of QTM. ${ }^{15}$ As shown in Figure 5, the relaxation time, $\tau$, for $\mathbf{1}$ is obviously longer than that of $\mathbf{2}$ at the same temperature. Obviously, the relaxation times for both complexes at the whole temperature region deviate from the Arrhenius law, indicating the presence of other relaxation pathways besides of Orbach processes. In fact, we found that the $\tau$ vs. $T$ data for both complexes can be properly fitted with an experimental $\tau^{-1} \sim T^{n}$ law (Figure 5) which corresponds to a Raman process. Values of $n=6.4$ and 7.2 were obtained for $\mathbf{1}$ and $\mathbf{2}$, respectively, indicating that a Raman process could also account for the entire relaxation behavior for both complexes.

Detailed ac susceptibility measurements were also performed on the polycrystalline samples of $\mathbf{2}$, obtained from the solid-state ligand substitution reaction of $\mathbf{1}$, to compare to the single crystal samples of $\mathbf{2}$ under the same external dc fields $\left(H_{\mathrm{dc}}=800 \mathrm{Oe}\right)$. As shown in Figure $\mathrm{S} 7$, the temperature- and frequency-dependent ac susceptibilities for the polycrystalline samples are very similar to the single crystal samples. Figure S8 shows that the temperature dependent magnetic relaxation time $(\tau)$ of polycrystalline samples of 2 matches well with the single-crystal samples, confirming the structural transformation from $\mathbf{1}$ to $\mathbf{2}$ through solid-state ligand substitution reaction.

$\boldsymbol{A b}$ initio calculation. The CASSCF calculated $g$-factors clearly show the large anisotropic character of these systems $\left(g_{x}, g_{y}, g_{z}\right.$ of $\left.1: 0.01,0.03,19.41 ; 2: 0.07,0.29,19.06\right)$. As reported in previous studies, ${ }^{16,17}$ the magnetic axiality of the ground Kramers' doublet plays a key role for the SMM performance of single-lanthanide complexes. As may be observed, complex 1 possesses a larger axial component $g_{z}$ and smaller transverse components $g_{\mathrm{x}}$ and $g_{\mathrm{y}}$ than 2; suggesting a weaker quantum tunneling of magnetization for the former. The different coordination environment of the Dy(III) center in both complexes entails a quite different direction of the magnetic moment for each of them (Figure 6 ). In the case of complex 1 , the magnetic moment seems to be aligned towards the axial oxygen atoms of two acac ligands, probably because of the large metal-ligand electron repulsion in that direction due to the negative charge of the ligands. ${ }^{5 \mathrm{~d}, 16}$ In comparison, the magnetic moment of complex 2 goes through the central carbon atom of one acac ligands. This arrangement seems to minimize the metal-ligand electron repulsion in the equatorial plane of the complex. These results translate into a noticeable distortion of the beta electron density obtained from the CASSCF ground state for both compounds, which takes a kind of oblate shape (Figure $6)^{5 a}$ The resulting magnetic anisotropy axis for both complexes shows a different electronic density distribution of the coordination atoms around the metal. As a consequence the geometry distortion breaks the $D_{4 \mathrm{~d}}$ symmetry, leading to a deviation of the ground-state magnetic anisotropy axis of Dy(III) ions from the $C_{4 \mathrm{v}}$ axis. A similar observation has also been reported in a recent study on bis(tetrapyrrole) dysprosium SMMs, where the easy axis of Dy(III) ions clearly deviates from the $C_{4 \mathrm{v}}$ axis by replacing one $\mathrm{N}$ atom of the porphyrin core by oxygen or sulfur atoms. ${ }^{6 \mathrm{~b}}$

Additionally, we also adopted the electrostatic model implemented in the MAGELLAN program which has been developed extensively in the $\left\{\mathrm{Dy}(\operatorname{acac})_{3}(\mathrm{~L})_{x}\right\}$ motifs to predict the orientation of the ground-state magnetic anisotropy axis. ${ }^{5 b}$ It is obvious that the directions of resulting electrostatic anisotropy axes for both complexes are very close to those from $a b$ initio calculations.
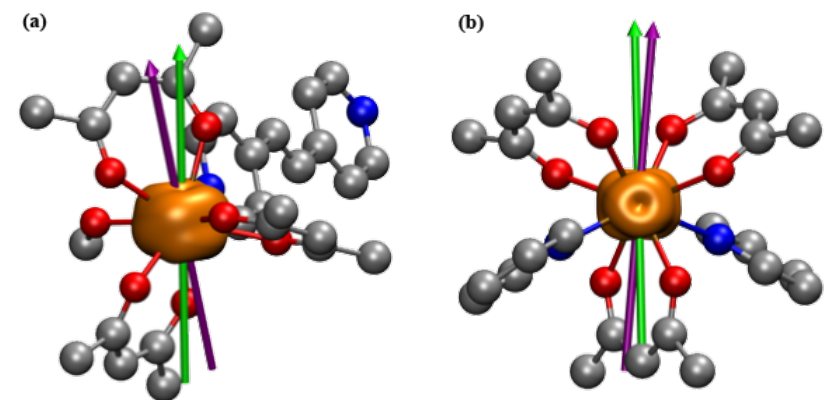

Figure 6 Beta spin density isosurface (orange) of the Dy(III) $f$ electrons found in the ab initio spin-free CASSCF calculation for complexes 1 (a) and 2 (b). The direction of the magnetic moment of the ground state obtained with MOLCAS and MAGELLAN are indicated as a green and a violet arrow, respectively. (Color code: $\mathrm{C}$, gray; $\mathrm{N}$, blue; $\mathrm{O}$, red; $\mathrm{H}$ atoms have been omitted for clarity).

The calculated energies of the lowest 3 Kramers' doublets (KDs) for both complexes are represented in Figure 7. The spin relaxation mechanisms indicate a plausible reversal of the magnetization through the first excited state because of an existing thermally assisted-quantum tunneling process, 
which should be more efficient for compound 2. In the case of this latter compound an Orbach relaxation process through the first KD could be also considered. Thus, the computed energy barriers enabling the spin relaxation should be 121.9 and $66.7 \mathrm{~cm}^{-1}$ for compounds 1 and 2 , respectively. For comparison, the effective energy barriers $\left(\Delta_{\text {eff }}\right)$ were extracted from the relaxation time data at the higher temperature region using an Arrhenius law. Values of $\Delta_{\text {eff }}=$ $71.7 \mathrm{~cm}^{-1}, \tau_{0}=3.37 \times 10^{-8} \mathrm{~s}$ for 1 and $\Delta_{\mathrm{eff}}=33.7 \mathrm{~cm}^{-1}, \tau_{0}=$ $1.39 \times 10^{-9} \mathrm{~s}$ for 2 were found (Figure S7). As may be observed, the $\Delta_{\text {eff }}$ values obtained by fitting the magnetic data are far from the energy gaps predicted by $a b$ inito calculation, further indicating that the magnetization reversal should be preferentially attributed to a Raman mechanism (Figure 5) rather to an Orbach process.
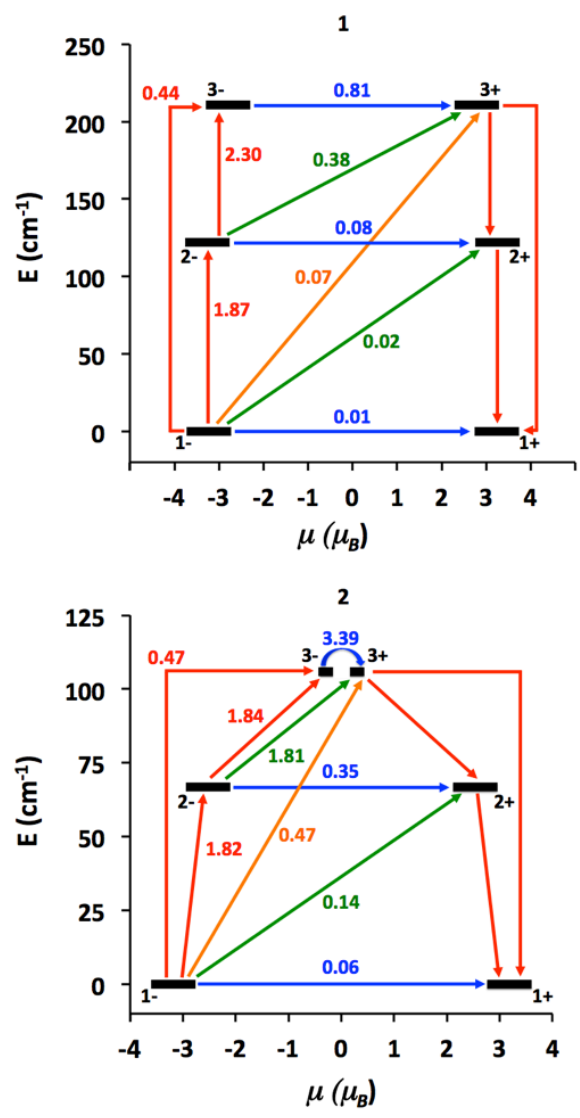

Figure 7 Lowest three Kramers doublets and $a b$ initio computed relaxation mechanism in $\mathbf{1}$ (up) and $\mathbf{2}$ (bottom). The thick black lines represent KDs as a function of their magnetic moment along the main anisotropy axis. Red lines indicate magnetization reversal mechanism. Blue lines correspond to ground state QTM and thermally assisted-QTM via the first and second excited KDs, green (and orange) lines show possible Orbach relaxation processes. The values indicated close to the arrows indicate the matrix elements of the transition magnetic moments (values higher than 0.1 provide efficient spin relaxation mechanisms). ${ }^{18}$

\section{CONCLUSIONS}

In conclusion, we have employed the solid-state ligand substitution reaction to transform the dinuclear Dy(III) compound $\left[\mathrm{Dy}_{2}(\mathrm{acac})_{6}(\mathrm{bpe})(\mathrm{MeOH})_{2}\right] \cdot \mathrm{bpe} \cdot 2 \mathrm{MeOH}$ (1) into the $\{\mathrm{Dy}\}_{n}$ chain compound [Dy(acac)3(bpe)] (2). This reaction takes advantage of the potential coordination ability of the guest bpe ligands of compound 1. ac susceptibility measurements for compound $\mathbf{2}$ samples, either from synthesis or after the solid-state ligand substitution reaction, confirm the effective transformation of $\mathbf{1}$ into $\mathbf{2}$. The rearrangement of the acac anions, methanol and bpe ligands around the Dy(III) centers in the solid-state transformation results in a remarkable change in the SMM behavior between both complexes. This work paves the way for fine-tuning of the magnetization dynamics of single-molecule magnets via a solid-state chemical substitution reaction.

\section{ASSOCIATED CONTENT}

Supporting Information. Powder X-ray diffraction patterns, selected bond lengths and angles, shape measures, thermogravimetric analysis and additional figures of structural and magnetic characterization. Crystallographic data in CIF format. This material is available free of charge via the Internet at http://pubs.acs.org.

\section{AUTHOR INFORMATION \\ Corresponding Author \\ *E-mail: liujliang5@mail.sysu.edu.cn; tongml@mail.sysu.edu.cn. \\ *E-mail: eliseo.ruiz@qi.ub.es.}

\section{Notes}

The authors declare no competing financial interest.

\section{ACKNOWLEDGMENT}

This work was supported by the "973 Project" (2014CB845602), the NSFC (Grant nos. 9142230221301197 and 2161101194) and the NSF of Guangdong (S2013020013002). The research reported was supported by the Spanish Ministerio de Economía y Competitividad (grant CTQ2015-64579-C3-1-P). E.R. thanks Generalitat de Catalunya for an ICREA Academia grant. J.J and E.R. thankfully acknowledge the computer resources, technical expertise and assistance provided by the CSUC.

\section{REFERENCES}

(1) (a) Sessoli, R.; Tsai, H. L.; Schake, A. R.; Wang, S.; Vincent, J. B.; Folting, K.; Gatteschi, D.; Christou, G.; Hendrickson, D. N. High-spin molecules: $\left[\mathrm{Mn}_{12} \mathrm{O}_{12}\left(\mathrm{O}_{2} \mathrm{CR}\right)_{16}\left(\mathrm{H}_{2} \mathrm{O}\right)_{4}\right]$. J. Am. Chem. Soc., 1993, 115, 1804-1816. (b) Sessoli, R.; Gatteschi, D.; Caneschi, A.; Novak, M. A. Magnetic bistability in a metal-ion cluster. Nature, 1993, 365, 141-143. (c) Leuenberger, M. N.; Loss, D. Quantum computing in molecular magnets. Nature, 2001, 410, 789-793.

(2) Gatteschi, D.; Sessoli, R.; Villain, J. Molecular Nanomagnets, Oxford University Press, New York, 2006.

(3) (a) Vincent, R.; Klyatskaya, S.; Ruben, M.; Wernsdorfer, W.; Balestro, F. Electronic read-out of a single nuclear spin using a molecular spin transistor. Nature, 2012, 488, 357-360. (b) Urdampilleta, M.; Klyatskaya, S.; Cleuziou, J-P.; Ruben, M.; Wernsdorfer, W. Supramolecular spin valves. Nat. Mater., 2011, 10, 502-506. (c) Ardavan, A.; Rival, O.; Morton, J. J. L.; Blundell, S. J.; Tyryshkin, A. M.; Timco, G. A.; Winpenny, R. E. P.; Will Spin-Relaxation Times in Molecular Magnets Permit Quantum Information Processing?. Phys. Rev. Lett., 2007, 98, 057201 .

(4) (a) Woodruff, D. N.; Winpenny, R. E. P.; Layfield, R. A.; 
Chem. Rev., 2013, 113, 5110-5148. (b) Liddle, S. T.; Slageren, J. V. Improving f-element single molecule magnets. Chem. Soc. Rev., 2015, 44, 6655-6669.

(5) (a) Rinehart, J. D.; Long, J. R. Exploiting single-ion anisotropy in the design of f-element single-molecule magnets. Chem. Sci., 2011, 2, 2078. (b) Chilton, N. F.; Collison, D.; McInnes, E. J. L.; Winpenny, R. E. P.; Soncini, A. An electrostatic model for the determination of magnetic anisotropy in dysprosium complexes. Nat. Commun., 2013, 4, 2551-2557. (c) Skomski, R. Simple Models of Magnetism, Oxford University Press, New York, 2008. (d) Chilton, N. F.; Langley, S. K.; Moubaraki, B.; Soncini, A.; Battena, S. R.; Murray, K. S. Single molecule magnetism in a family of mononuclear $\beta$-diketonate lanthanide(III) complexes: rationalization of magnetic anisotropy in complexes of low symmetry. Chem. Sci., 2013, 4, 1719-1730. (e) Ungur, L.; Chibotaru, L. F. Ab Initio Crystal Field for Lanthanides. Chem. Eur. J., 2017, 23, 3708-3718.

(6) (a) Wu, J.; Jung, J.; Zhang, P.; Zhang, H.; Tang, J.; Guennic, B. L. Cis-trans isomerism modulates the magnetic relaxation of dysprosium single-molecule magnets. Chem. Sci., 2016, 7, 3632-3639. (b) Cao, W.; Gao, C.; Zhang, Y.-Q.; Qi, D.; Liu, T.; Wang, K.; Duan, C.; Gao, S.; Jiang, J. Rational enhancement of the energy barrier of bis(tetrapyrrole) dysprosium SMMs via replacing atom of porphyrin core. Chem. Sci., 2015, 6, 5947-5954. (c) Liu, J.-L.; Chen, Y.-C.; Zheng, Y.-Z.; Lin, W.-Q.; Ungur, L.; Wernsdorfer, W.; Chibotaru, L. F.; Tong, M.-L. Switching the anisotropy barrier of a single-ion magnet by symmetry change from quasi- $D_{5 \mathrm{~h}}$ to quasi- $O_{\mathrm{h}}$. Chem. Sci., 2013, 4, 3310-3316. (d) Sun, W.-B.; Yan, P.-F.; Jiang, S.-D.; Wang, B.-W.; Zhang, Y.-Q.; Li, H.-F.; Chen, P.; Wang, Z.-M.; Gao, S. High symmetry or low symmetry, that is the question high performance Dy(III) single-ion magnets by electrostatic potential design. Chem. Sci., 2016, 7, 684-691. (e) Zhang, X.-J.; Vieru, V.; Feng, X.-W.; Liu, J.-L., Zhang, Z.J.; Na, B.; Shi, W.; Wang, B.-W.; Powell, A. K.; Chibotaru, L. F.; Gao, S.; Cheng, P.; Long, J. R. Influence of Guest Exchange on the Magnetization Dynamics of Dilanthanide Single-Molecule Magnet Nodes within a Metal-Organic Framework. Angew. Chem. Int. Ed., 2015, 34, 9861-9865. (f) Liu, J.-L.; Wu, J.-Y.; Huang, G.-Z.; Chen, Y.-C.; Jia, J.-H.; Ungur, L.; Chibotaru, L. F.; Chen, X.-M.; Tong, M.-L. Desolvation-Driven 100-Fold Slow-down of Tunneling Relaxation Rate in Co (II)-Dy (III) Single-Molecule Magnets through a Single-Crystal-to-Single-Crystal Process, Sci. Rep., 2015, 5, 16621.

(7) Jiang, S.-D.; Wang, B.-W.; Su, G.; Wang, Z.-M.; Gao, S. A Mononuclear Dysprosium Complex Featuring Single-Molecule-Magnet Behavior. Angew. Chem. Int. Ed., 2010, 49, 7448-7451.

(8) (a) Bi, Y.; Guo, Y.-N.; Zhao, L.; Guo, Y.; Lin, S.-Y.; Jiang, S.-D.; Tang, J.; Wang, B.-W.; Gao, S. Capping Ligand Perturbed Slow Magnetic Relaxation in Dysprosium Single-Ion Magnets. Chem. Eur. J., 2011, 17, 12476-12481. (b) Chen, G.-J.; Guo, Y.-N.; Tian, J.-L.; Tang,J.; Gu, W.; Liu, X.; Yan, S.-P.; Cheng, P.; Liao, D.-Z. Enhancing Anisotropy Barriers of Dysprosium(III) Single-Ion Magnets. Chem. Eur. J., 2012, 18, 2484-2487. (d) Zhang, S.; Ke, H.; Sun, L.; Li, X.; Shi, Q.; Xie, G.; Wei, Q.; Yang, D.; Wang, W.; Chen, S. Magnetization Dynamics Changes of Dysprosium(III) Single-Ion Magnets Associated with Guest Molecules. Inorg. Chem. 2016, 55, 3865-3871.

(9) SHELXTL 6.10, Bruker Analytical Instrumentation, Madison, Wisconsin (USA), 2000.

(10) Aquilante, F.; Vico, L. D.; Ferre, N.; Ghigo, G.; Malmqvist, P. A.; Neogrady, P.; Pedersen, T. B.; Pitonak, M.;
Reiher, M.; Roos, B. O.; Serrano-Andres, L.; Urban, M.; Veryazov, V.; Lindh, R. MOLCAS 7: The Next Generation. $J$. Comput. Chem., 2010, 31, 224-247.

(11) (a) Roos, B. O.; Veryazov, V.; Widmark, P.-O. Relativistic atomic natural orbital type basis sets for the alkaline and alkaline-earth atoms applied to the ground-state potentials for the corresponding dimers. Theor. Chim. Acta, 2004, 111, 345-351. (b) Roos, B. O.; Lindh, R.; Malmqvist, P.-Å.; Veryazov, V.; Widmark, P.-O. Main Group Atoms and Dimers Studied with a New Relativistic ANO Basis Set. J. Phys. Chem. A 2004, 108, 2851-2858. (c) Roos, B. O.; Lindh, R.; Malmqvist, P.-Å.; Veryazov, V.; Widmark, P.-O. New Relativistic ANO Basis Sets for Transition Metal Atoms. J. Phys. Chem. A 2005, 109, 6575-6579. (d) Roos, B. O.; Lindh, R.; Malmqvist, P.-Å.; Veryazov, V.; Widmark, P.-O. New relativistic ANO basis sets for actinide atoms. Chem. Phys. Lett. 2005, 409, 295-299. (e) Ungur, L.; Chibotaru, L. F.; Magnetic anisotropy in the excited states of low symmetry lanthanide complexes. Phys. Chem. Chem. Phys., 2011, 13, 20086-20090. (f) Chibotaru, L. F.; Ungur, L.; $\mathrm{Ab}$ initio calculation of anisotropic magnetic properties of complexes. I. Unique definition of pseudospin Hamiltonians and their derivation. J. Chem. Phys., 2012, 137, 064112.

(12) (a) Chibotaru, L. F.; Ungur, L.; Soncini, A. The Origin of Nonmagnetic Kramers Doublets in the Ground State of Dysprosium Triangles: Evidence for a Toroidal Magnetic Moment. Angew. Chem. Int. Ed. 2008, 47, 4126-4129. (b) Chibotaru, L. F.; Ungur, L.; Aronica, C.; Elmoll, H.; Pillet, G.; Luneau, D. Structure, Magnetism, and Theoretical Study of a Mixed-Valence $\mathrm{Co}_{3}{ }_{3} \mathrm{Co}^{\mathrm{III}}{ }_{4}$ Heptanuclear Wheel: Lack of SMM Behavior despite Negative Magnetic Anisotropy. J. Am. Chem. Soc. 2008, 130, 12445-12455.

(13) (a) Alvarez, S.; Alemany, P.; Casanova, D.; Cirera, J.; Llunell, M.; Avnir, D. Shape maps and polyhedral interconversion paths in transition metal chemistry. Coord. Chem. Rev., 2005, 249, 1693-1708; (b) Casanova, D.; Llunell, M.; Alemany, P.; Alvarez, S. The Rich Stereochemistry of Eight-Vertex Polyhedra: A Continuous Shape Measures Study. Chem. Eur. J., 2005, 11, 1479-1494.

(14) (a) Bradshaw, D.; Warren, J. E.; Rosseinsky, M. J. Reversible Concerted Ligand Substitution at Alternating Metal Sites in an Extended Solid. Science, 2007, 315, 977-980. (b) Wöhlert, S.; Boeckmann, J.; Wriedt, M.; Näther, C. Coexistence of Metamagnetism and Slow Relaxation of the Magnetization in a Cobalt Thiocyanate 2D Coordination Network. Angew. Chem. Int. Ed., 2011, 50, 6920-6923.

(15) (a) Gregoli, L.; Danieli, C.; Barra, A. L.; Neugebauer, P.; Pellegrino, G.; Poneti, G.; Sessoli, R.; Cornia, A. Magnetostructural Correlations in Tetrairon(III) Single-Molecule Magnets. Chem. Eur. J. 2009, 15, 6456-6467; b) Kajiwara, T.; Takahashi, K.; Hiraizumi, T.; Takaishi, S.; Yamashita, M. Coordination enhancement of single-molecule magnet behavior of $\mathrm{Tb}(\mathrm{III})-\mathrm{Cu}(\mathrm{II})$ dinuclear systems. Polyhedron 2009, 28, 1860-1863. (c) Bernot, K.; Pointillart, F.; Rosa, P.; Etienne, M.; Sessoli, R.; Gatteschi, D. Single molecule magnet behaviour in robust dysprosium-biradical complexes. Chem. Commun. 2010, 46, 6458-6460.

(16) Aravena, D.; Ruiz, E. Shedding Light on the Single-Molecule Magnet Behavior of Mononuclear DyIII Complexes. Inorg. Chem., 2013, 52, 13770-13778.

(17) (a) Chibotaru, L. F.; Ungur, L.; Aronica, C.; Elmoll, H.; Pilet, G.; Luneau, D. Structure, Magnetism, and Theoretical Study of a Mixed-Valence $\mathrm{Co}_{3}{ }_{3} \mathrm{Co}^{\mathrm{III}}{ }_{4}$ Heptanuclear Wheel: Lack of SMM Behavior despite Negative Magnetic Anisotropy. J. Am. Chem. Soc. 2008, 130, 12445-12455. (b) Feltham, H. L. C.; Lan, Y.; Klöwer, F.; Ungur, L.; Chibotaru, L. F.; Powell, A. K.; 
Brooker, S. A Non-sandwiched Macrocyclic Monolanthanide Single-Molecule Magnet: The Key Role of Axiality. Chem. Eur. J. 2011, 17, 4362-4365.

(18) Gómez-Coca, S.; Aravena, D.; Morales, R.; Ruiz, E. Large magnetic anisotropy in mononuclear metal complexes. Coord. Chem. Rev., 2015, 289-290, 379-392. 
TOC

A Dy(III) single-molecule magnet undergoes a structural transformation from a dinuclear complex to a one-dimensional chain upon solid-state ligand substitution reaction, which further varies the slow magnetic relaxation behavior.

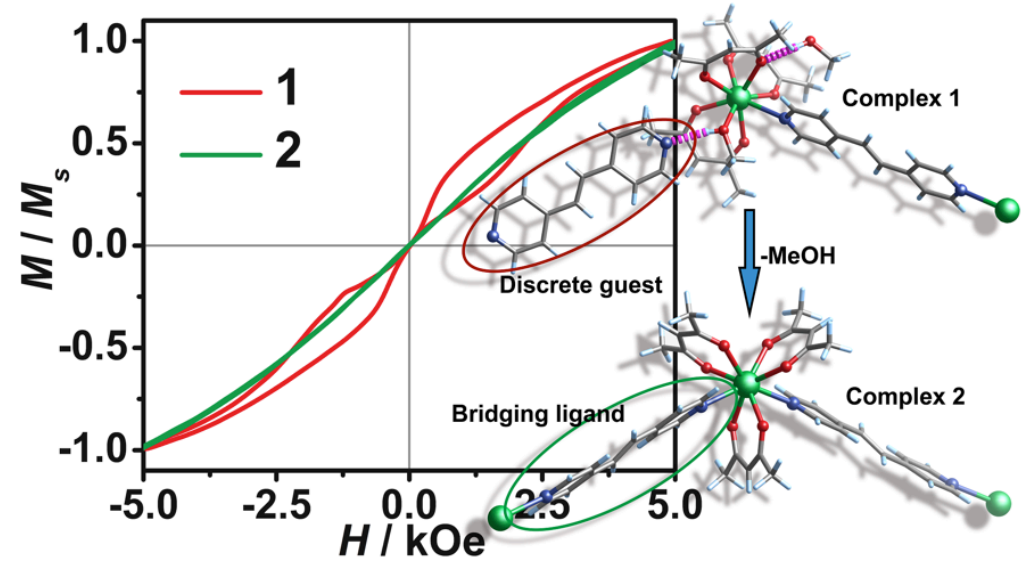

